\title{
Aplicação do earned value em projetos complexos - um estudo de caso na EMBRAER
}

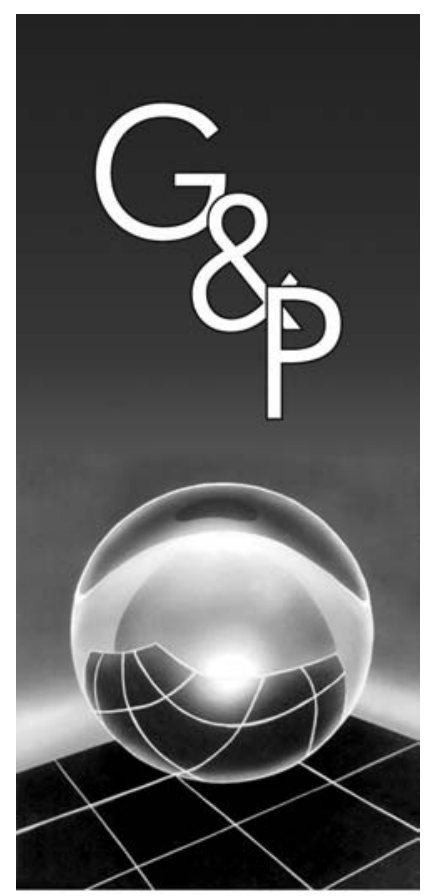

\author{
Rogério Abdala Giacometti \\ Carlos Eduardo Sanches da Silva \\ Helder José Celani de Souza \\ Fernando Augusto Silva Marins \\ Elizabete Ribeiro Sanches da Silva
}

Resumo

O gerenciamento do Earned Value vem obtendo aceitação crescente nas empresas e entre profissionais tais como gerentes de projetos e funcionais, devido à sua efetividade no controle dos resultados do projeto. Earned Value é definido como uma ferramenta de controle que permite avaliar simultaneamente de maneira quantitativa como se encontram os custos e prazos em uma data de controle, permitindo também prever a data de término e o valor gasto (VARGAS, 2005). Este artigo avalia a aplicabilidade do earned value como ferramenta de controle de projetos aeronáuticos na EMBRAER. Contribui também para a aplicação do earned value no controle de projetos complexos. Utilizam-se como técnicas de coleta de dados: entrevistas, observações e análise documental. Conclui-se que o earned value é utilizado apenas nos projetos da área de aviação de defesa, por ser uma exigência contratual. $O$ uso do earned value nas áreas de programas executivos e comerciais restringe-se às fases de definição conjunta, projeto detalhado e certificação, pois o escopo do projeto é bem definido, o cronograma é detalhado e os custos são previstos e obtidos com maior acuidade. Nas demais fases de desenvolvimento do produto, o uso do earned value é restrito, devido principalmente às constantes alterações de escopo do produto e do projeto.

Palavras-chave: Projetos. Controle. Earned value.

\section{Contexto da pesquisa}

Muitos executivos concordam que para solucionar a maioria dos problemas corporativos é necessário obter melhor controle e otimizar a alocação dos recursos existentes. Com o intuito de obter melhor desempenho na solução destes novos problemas que vêm surgindo, cada vez mais executivos procuram mecanismos que auxiliem no processo decisório. Para isso, a gestão de projeto é um dos meios a serem considerados (KERZNER, 2005).

Define-se projeto como esforço temporário empreendido para criar um único produto, serviço ou resultado (PMBOK, 2004). Cada produto ou serviço é diferente em alguma característica dentre os vários produtos e serviços similares (ROZENES et al., 2004).

Em relação aos negócios de uma organização, dada à importância relativa ou predominância de atividades rotineiras ou de projetos, as organizações podem ser orientadas para a rotina, orientadas para projetos e para as híbridas. Deve-se, no entanto, considerar que haverá, em todas as organizações, a ocorrência de operações rotineiras e de projetos. Dependendo do tipo de negócio, as organizações estabelecem estruturas organizacionais que determinam a forma de atuação e a disponibilidade de recursos alocados. As organizações podem ter estruturas funcionais, por projetos e matriciais (GOMES, 2004). Independentemente do negócio e da estrutura organizacional, existem projetos e a necessidade de serem gerenciados e terem seu desempenho periodicamente avaliado. Bowman (2005) reforça a necessidade de controle, citando que uma importante decisão, quando estamos gerenciando a execução de um projeto, é o controle das atividades e seus custos, que possuem duração específica e custo específico dentro do contexto do projeto.

Gestão de Projetos deve direcionar as organizações a utilizarem as melhores informações disponíveis por meio de ferramentas inovadoras e técnicas que se adaptem às suas condições (KWAK; WATSON, 2004). 
O sucesso da gestão de um projeto acontece quando o projeto é finalizado dentro do prazo, com o menor custo possível, com a melhor qualidade, sem acidentes, etc. Em outras palavras, pode ser refletido por indicadores de custo, prazo, qualidade, produtividade, consumo de matéria-prima, perdas, etc., numa condição ótima. Um sistema de controle é um importante elemento na gestão de projeto. Um sistema de controle permite encontrar discrepâncias entre o planejado e o realizado e cabe ao gerente de projeto e à sua equipe identificar as causa dos desvios e tomar decisões para colocar o projeto dentro dos valores planejados (NAVON, 2005).

A gestão de projetos engloba atividades complexas e dinâmicas e envolve múltiplas variáveis com relações não lineares. Enquanto os problemas encontrados ao longo da execução de um projeto forem tratados de maneira estática, atrasos e custos não desejados ocorrerão. Tal situação entra em contradição com equipamentos de alta tecnologia e técnicas avançadas de gestão. Para evitar estes resultados indesejáveis, enormes esforços vêm sendo realizados no planejamento e controle de projetos (LEE et al., 2005).

$\mathrm{O}$ earned value é uma técnica de controle que permite avaliar, em qualquer momento, a performance de prazos, custos e escopo do projeto: comparando os prazos planejados para o cumprimento de tarefas (work schedule) com as tarefas efetivamente realizadas (earned value) e também dentro da perspectiva de custos planejados $x$ custos efetivamente incorridos (ROVAI; TOLEDO, 2002).

$\mathrm{Na}$ literatura encontram-se outros nomes para a técnica earned value, tais como earned value management system (ROVAI; TOLEDO, 2002), earned value management (KIM, 2000). Nesta pesquisa, adotaremos apenas earned value, por ser o nome mais difundido segundo Fleming e Koppelman (2000). A tradução do termo earned value tem sido equivocadamente escrita como "valor agregado" (OLIVEIRA, 2003; VARGAS, 2005). Vale destacar que o conceito de valor agregado engloba a lucratividade, o que não ocorre com o earned value.

Willians (2002) diz que a análise de earned value se baseia em parâmetros empregados, usando somente métodos tradicionais. Naturalmente, se o trabalho terminado tiver sido significativamente superestimado, ou se os processos estabelecidos no projeto gerarem um trabalho extra-significativo, os cálculos do earned value serão inúteis ou pouco aproveitados. Assim, um projeto que faz o uso significativo de earned value pode ser ajudado fortemente se tiver disponível um modelo que descreva os efeitos sistêmicos. Um modelo sistêmico pode dar estimativas de custo e de duração na conclusão, podendo ser aplicada uma triangulação valiosa com a análise de earned value, gerando uma diferença relevante ao tipo de modelo casual.
De acordo com Vargas (2005), a Análise de Earned Value apresenta um conjunto de recursos intrínsecos poderoso, abrangente e variado, tais como projeção de pagamentos e planejamento de resultados. Encontra-se, porém, notável dificuldade tanto na coleta dos dados quanto na baixa velocidade da geração da informação.

A utilização do earned value como ferramenta de controle está ganhando aceitação elevada entre os gerentes sênior e os gerentes de projeto, que vêm percebendo que os problemas do earned value estão diminuindo e as utilidades estão melhorando. Esta aceitação elevada é encontrada no setor público e privado, sugerindo que não é apenas uma ferramenta para projetos grandes no setor público (KIM et al., 2003).

Podem-se identificar várias pesquisas que abordam o gerenciamento de projetos (KERZNER, 2005; ROZENES et al., 2004; KAWK; WATSON, 2004; NAVON, 2005; BOWMAN, 2005; LEE et al., 2005; VARGAS, 2005; KIM, 2003) e o controle de custos e prazos pelo earned value (KERZNER, 2005; MARTINS, 2001; WILLIANS, 2002; VARGAS, 2005). Conforme aumenta a complexidade de um projeto (por exemplo, uma aeronave) surge a necessidade de se avaliar o uso do earned value nas fases do programa de desenvolvimento de produtos (DIP).

Shenhar e Wideman (2000) relatam em sua pesquisa, baseada em mais de 120 projetos, que com o aumento de incerteza se faz necessário um aumento da administração técnica e, da mesma forma, com um aumento de complexidade se faz necessária maior ênfase em gerenciamento de projetos (Figura 1).

Para Maximiano (2002), a complexidade de uma situação é medida pelo número de variáveis que contém e, assim, um projeto complexo é aquele que apresenta grande número de variáveis a serem administradas. Complementa Oliveira (2003) que a complexidade de um projeto é ligada ao seu porte, mas este não é o único fator determinante, podem existir outros fatores (por exemplo: a diversidade cultural entre os envolvidos no projeto, o nível tecnológico agregado ao projeto, o grau de inovação, restrições, alterações de escopo, prazos longos, número de especialidades envolvidas). Com o aumento da complexidade o sucesso depende do controle. Embora o gerenciamento de projetos possa ser usado em qualquer projeto, nos projetos complexos, além da aplicabilidade, existe a real necessidade de um gerenciamento específico.

Existe uma quantidade muito grande de projetos que são finalizados com atrasos e sobre-custos, ou até mesmo não são finalizados (em geral pelos mesmos motivos). Uma alegação freqüente por parte dos gestores é a de que estes só conseguem perceber a magnitude real de tais problemas em estágios já avançados de progresso, quando, na maioria das vezes, já não conseguem tomar ações corretivas em tempo de evitar o fracasso do projeto. 


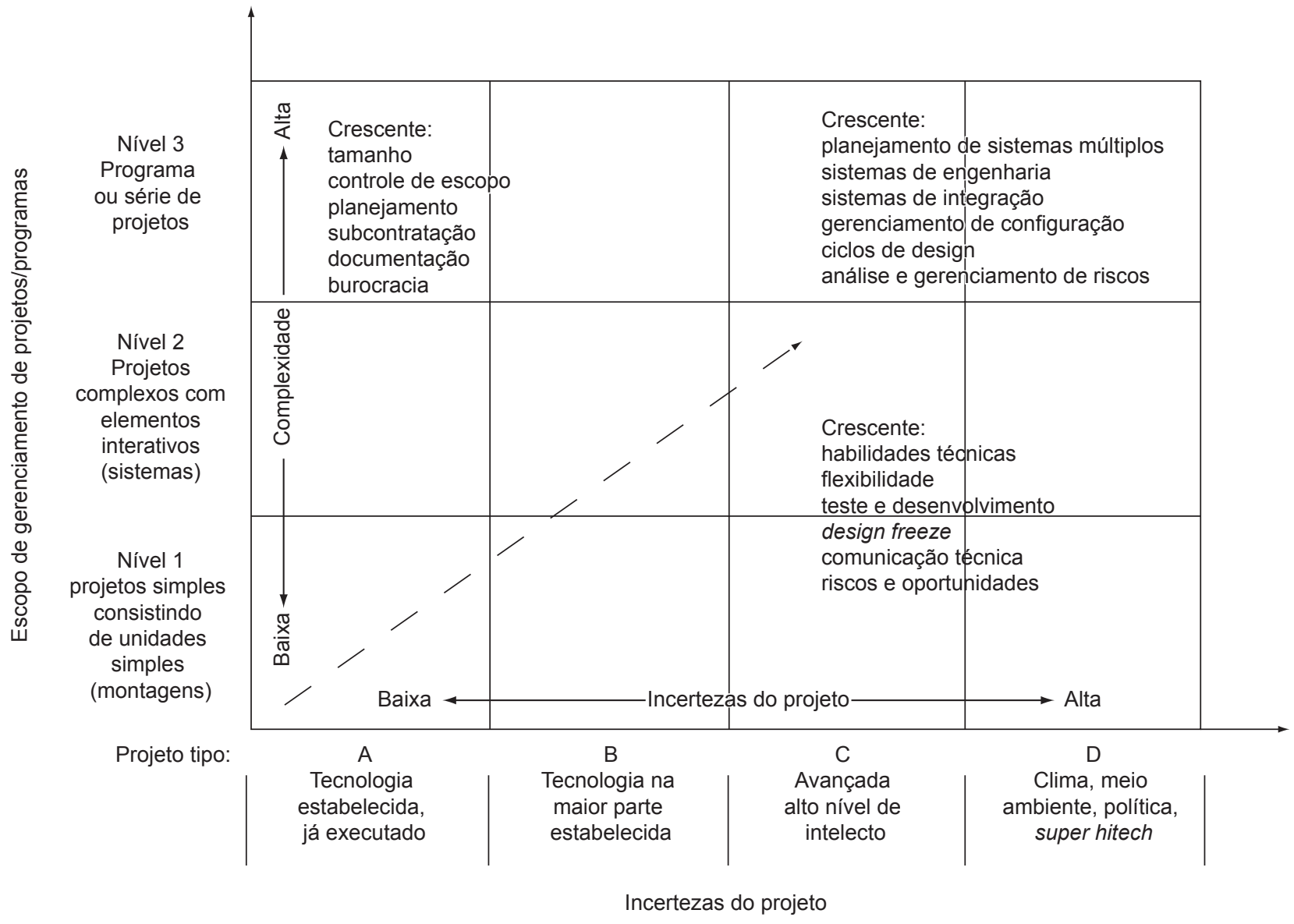

Figura 1. Classificação de projetos por complexidade x incertezas (SHENHAR; WIDEMAN, 2000).

A importância do earned value advém do fato de poder oferecer diagnóstico preciso e completo dos prazos e custos em qualquer fase do projeto (OLIVEIRA, 2003).

Välimãki (1995) e Fleming e Koppelman (2000) advogam que a verdadeira gestão de projetos obrigatóriamente deve ter em seu âmago os princípios do Earned Value, pois este é o meio mais eficiente de se ter controle e visualização sobre o progresso do projeto.

Surge neste contexto a seguinte questão desta pesquisa: Como o earned value se aplica nas etapas do DIP como técnica de controle de projetos complexos?

\section{Earned value}

Os projetos militares da Segunda Guerra Mundial avançaram no campo da gerência de projeto. Em 1967, o departamento de Defesa dos Estados Unidos liberou sua primeira lista oficial de critérios dos sistemas de controle (C/SCSC - cost/schedule control systems criteria), sinalizando a iniciação formal da análise de earned value, que representa ainda a melhor possibilidade de se "medir" projetos de uma maneira integrada (CIOFFI, 2006).
Na época de sua criação, o C/SCSC tornou-se obrigatório para todos os contratos e projetos das forças armadas americanas. Isto, por um lado, ajudou a difundir o earned value, porém a sua imposição, aliada à grande burocracia gerada, acabou estigmatizando o seu uso e fazendo com que o critério fosse visto como um mero sistema de se reportar ao cliente e não como uma ferramenta útil de controle (FLEMING; KOPPELMAN, 2000).

No início da década de 90 , a análise de earned value ressurgiu no ambiente administrativo com força total, juntamente com o Gerenciamento de Projetos e, desde então, muitos estudos vêm sendo realizados a respeito desta técnica, o que indica que ela permanecerá por um bom tempo em voga nos meios acadêmicos e profissionais (OLIVEIRA, 2003).

Embora esteja enraizada dentro da área de Gerenciamento de Custos, ela sofre ou exerce influência em quase todas as áreas da gestão do projeto, uma vez que seu princípio se baseia na inter-relação entre custos, prazos e escopo. Elementos que figuram em todas as áreas de atuação do Gerente de Projetos (OLIVEIRA, 2003). 
Vargas (2005) descreve a sistemática do earned value na Figura 2.

Na Figura 2, tem-se a curva referente à linha de base do orçamento (BCWS) que percorre todo o período planejado do projeto, a curva dos custos reais incorridos (ACWP - actual cost of work performed) e a curva do valor que foi agregado até uma determinada data (BCWP - budget cost of work performed). Em um caso ideal, a curva de custos reais (ACWP) e a curva de earned value (BCWP) deveriam se sobrepor exatamente à curva do orçamento. Isto representaria um desempenho de custos e prazos $100 \%$ igual ao planejado para o projeto. Sabendo, entretanto, que isto é muito difícil de ocorrer na realidade, a idéia é que se considere sempre a curva do orçamento (BCWS) como uma referência e um objetivo a ser perseguido, tanto em termos de custos como de prazos.
A análise crítica sobre o progresso e desempenho do projeto é possível pelas correlações entre os parâmetros citados, ao longo do período do projeto.

a) CV (cost variance ou variação nos custos): é a diferença entre o valor que foi fisicamente agregado (BCWP) e o custo real do projeto (ACWP), até uma determinada data. Se CV for positiva, a atividade estará com custo abaixo do valor previsto; se for negativa, a atividade terá ultrapassado o orçamento, até a referida data.

$$
\mathrm{CV}=\mathrm{BCWP}-\mathrm{ACWP}
$$

b) SV (schedule variance ou variação no cronograma): é a diferença, em termos de custo, entre o earned value (BCWP) e o valor planejado (BCWS). Se SV for positiva, o projeto está adiantado; se for negativa, o projeto está atrasado.

$$
\mathrm{SV}=\mathrm{BCWP}-\mathrm{BCWS}
$$

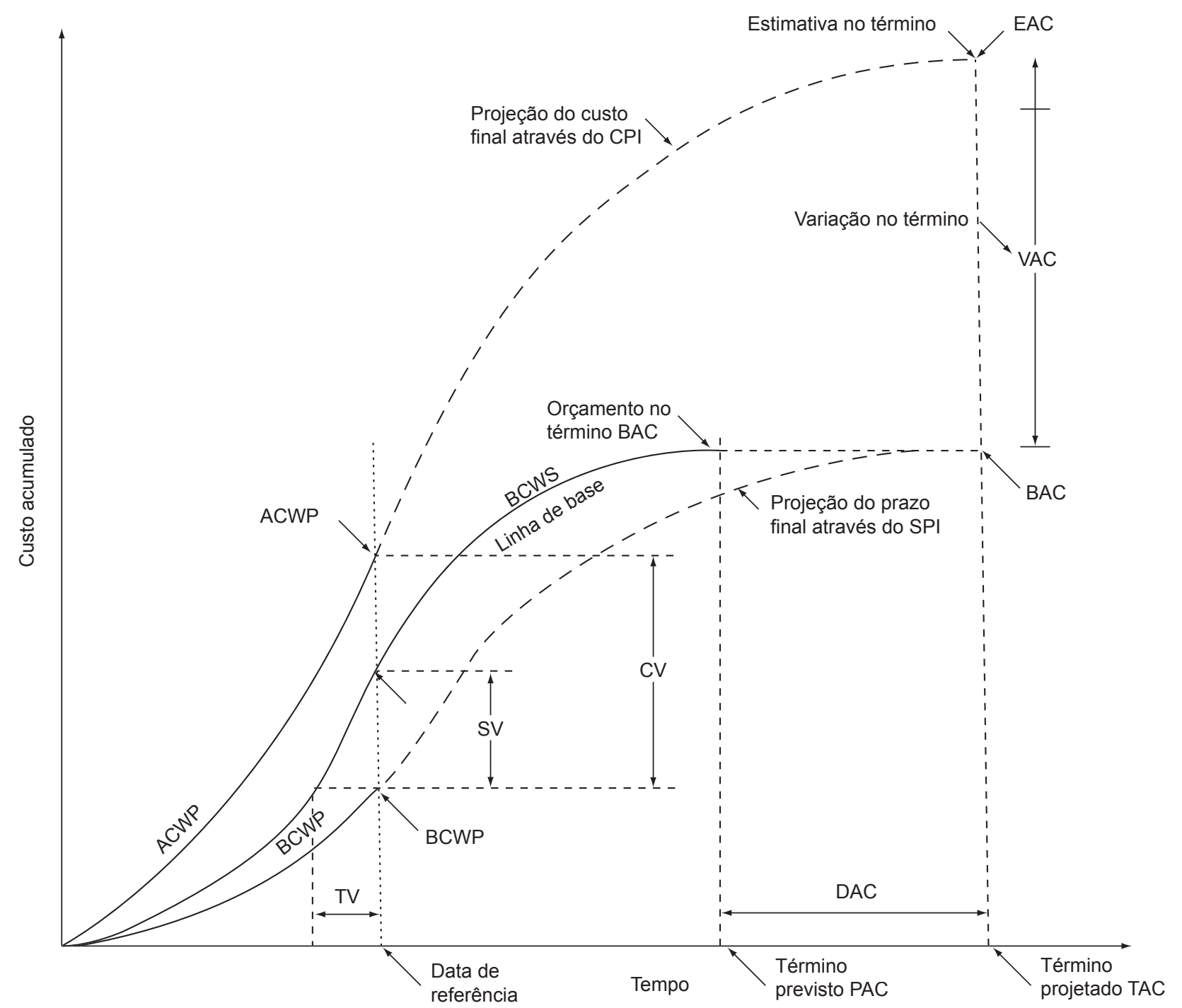

Figura 2. Análise do earned value com projeções e tendências dos prazos finais e custos finais (VARGAS, 2005). 
c) TV (time variance ou variação no tempo): é a diferença, em termos de tempo, entre o earned value (BCWP) e o valor planejado (BCWS). É encontrado graficamente pela projeção da curva de BCWP, encontrando a data em que o BCWS agrega o mesmo valor de BCWP. A diferença entre a data de referência e a data em que BCWS agrega o mesmo valor que BCWP representa o atraso ou adiantamento do projeto.

d) VAC (variation at completion ou variação final dos custos): é a diferença entre o custo orçado (BAC budget at completion) e o custo projetado final (EAC - estimated at completion).

$$
\mathrm{VAC}=\mathrm{BAC}-\mathrm{EAC}
$$

e) DAC (delay at completion ou variação final dos prazos): é a diferença entre o término projetado (TAC - time at completion) do projeto e o Término Previsto Originalmente (PAC - planned at completion).

$$
\mathrm{DAC}=\mathrm{TAC}-\mathrm{PAC}
$$

f) SPI (schedule performance index ou índice de performance de cronograma): é a relação entre o earned value (BCWP) e o valor planejado (BCWS) em uma determinada data. O SPI mostra a taxa de conversão do valor previsto em earned value até a referida data.

$$
\mathrm{SPI}=\frac{\mathrm{BCWS}}{\mathrm{BCWP}}
$$

Como exemplo, temos que um SPI $=0,80$ indica que $80 \%$ do tempo previsto no orçamento foi convertido em trabalho. Isto resulta em uma perda de $20 \%$ no tempo disponível. Resumindo, pode-se generalizar que um SPI igual a 1 indica que o valor planejado foi integralmente agregado ao projeto. Se o SPI for menor que 1, o projeto está atrasado. Se o SPI for superior a 1, o projeto está adiantado.

CPI (cost performance index ou índice de performance de custo): é a relação entre o earned value (BCWP) e o custo real do projeto (ACWP). O CPI mostra a taxa entre os valores reais consumidos e os valores agregados no mesmo período.

$$
\mathrm{CPI}=\frac{\mathrm{ACWP}}{\mathrm{BCWP}}
$$

Como exemplo, temos que um CPI $=0,80$ indica que para $\$ 1$ de capital consumido, apenas \$ 0,80 estão sendo convertidos fisicamente em produto. A perda, portanto, é de \$ 0,20 por \$1 gasto. Analogamente ao SPI, um CPI igual a 1 indica que o valor gasto pelo projeto foi integralmente agregado ao projeto (o projeto está dentro do orçamento previsto). Se o CPI for menor que 1 , o projeto está gastando mais do que o previsto (provavelmente haverá sobre-custo ao final do projeto). Se o CPI é superior a 1, o projeto está custando abaixo do orçamento previsto.

Pode-se observar que o planejamento de um projeto para a utilização da análise do earned value consiste na realização de quatro passos (VARGAS, 2005):

g) definir o escopo de projeto por meio de uma estrutura analítica do projeto (EAP) ou qualquer outro tipo de estrutura de modo a definir com precisão o trabalho a ser realizado;

h) criar cronograma do projeto de modo a identificar a distribuição das atividades no tempo;

i) alocar os recursos nas atividades e calcular o orçamento de cada um dos pacotes de trabalho do projeto, com base na sua duração e carga de trabalho dos recursos atribuídos; e

j) estabelecer uma previsão-base (baseline) de custos e prazos que constituirá o conjunto de valores para o projeto (BCWS).

$\mathrm{O}$ earned value tem demonstrado ser uma das mais eficazes ferramentas técnicas utilizadas na gestão de projetos, sua grande virtude é proporcionar ao gerente de projetos uma advertência prévia acerca do desempenho do projeto. Já na fase inicial do projeto, com aproximadamente 10 ou $15 \%$ de tarefas concluídas, torna-se possível avaliar se o projeto está cumprindo as tarefas do cronograma no prazo planejado, dentro do orçamento aprovado e se não há nenhum desvio de escopo (ROVAI; TOLEDO, 2002).

No que diz respeito ao valor da técnica, os resultados encontrados para Análise de earned value são enquadrados em uma faixa de pouco valor (Quadro 1), ficando

Quadro 1. Técnicas de gerenciamento de projetos (THAMHAIN, 1998).

\begin{tabular}{lcc}
\hline \multicolumn{1}{c}{ Técnica } & $\begin{array}{c}\text { Popularidade } \\
(\boldsymbol{\%})\end{array}$ & $\begin{array}{c}\text { Valor da } \\
\text { técnica }\end{array}$ \\
\hline Controle de prazos & 99 & 3,25 \\
Definição do projeto & 98 & 3,75 \\
Revisão de projeto & 93 & 3,15 \\
Controle de orçamentos & 92 & 3,25 \\
Revisão de design & 87 & 3,50 \\
Prototipação & 82 & 3,25 \\
Verificação de status & 82 & 3,75 \\
Relatório de deficiências & 68 & 2,50 \\
Relatório de ações & 65 & 3,00 \\
Análise de requerimentos & 52 & 3,20 \\
Benchmarking & 52 & 1,50 \\
PERT/COM & 42 & 1,50 \\
Análise de earned value & 41 & 1,75 \\
Análise do caminho crítico & 32 & 2,00 \\
QFD - quality function deployment & 28 & 2,00 \\
Análise de compressão de duração & 18 & 1,00 \\
\hline
\end{tabular}


abaixo de praticamente todas as técnicas analisadas, o que sugere que a popularidade da técnica não retrata sua aplicabilidade ou valor (THAMHAIN, 1998).

$\mathrm{Na}$ tentativa de justificar o baixo valor, Thamhain (1998) afirma que a pouca aplicabilidade pode ser atribuída às diferentes barreiras, sejam elas internas ou do ambiente. São elas: falta de compreensão do funcionamento da técnica; ansiedade quanto ao uso adequado da ferramenta; utilização da ferramenta requerendo muito trabalho e consumindo tempo; ferramentas limitando a criatividade no uso das estratégias; inconsistência da ferramenta com os procedimentos gerenciais/processos de negócios; métodos de controle atuando como ameaçadores, no que diz respeito à liberdade da equipe; o propósito e seu benefício muitas vezes são vagos e imprecisos; custo de sua implementação considerado elevado; o trabalho não é conduzido em equipe; equipe sempre ocupada para aprender novas ferramentas; não atuando como ferramentas-controle e, sim, como ferramentas justificadoras de eventuais atrasos e desvios; experiência anterior fracassada na utilização de outras técnicas; e desconforto com a pouca familiaridade da técnica.

Os principais fatores para uma boa aceitação do earned value, segundo Kim et al. (2003), são: a aproximação da alta gerência com a ferramenta; estabelecimento de uma cultura voltada para projeto por parte dos gerentes funcionais e suas lideranças subseqüentes, para persuadir membros da equipe de projeto a usar o earned value; flexibilidade aos gerentes funcionais e de projeto e aos trabalhadores ou grupos para selecionar seu próprio formulário do uso do earned value, dentro uma estrutura de diretrizes gerais; fornecer o treinamento para os usuários do earned value. Após algum tempo, com a experiência adquirida na organização, em combinação com o treinamento, a aceitação será atingida.

De acordo com Vargas (2005) a análise de earned value apresenta um conjunto de recursos intrínsecos poderosos, abrangentes e variados, tais como projeção de pagamentos e previsibilidade. Porém, encontra-se notável dificuldade, tanto na coleta dos dados quanto na baixa velocidade da geração da informação. Complementa o autor, com certo exagero, que em média os custos de se implementar a Análise de earned value se situam em torno de $1 \%$ a $1,5 \%$ dos custos do projeto (VARGAS, 2005).

\section{Estudo de caso EMBRAER}

O universo de estudo escolhido para este trabalho foi a Empresa Brasileira de Aeronáutica (EMBRAER) por possuir o desenvolvimento de projetos complexos, por utilizar a ferramenta de earned value e pela facilidade de acesso aos dados.
A EMBRAER foi a maior exportadora brasileira entre os anos de 1999 e 2001, e foi a segunda maior empresa exportadora nos anos de 2002, 2003 e 2004. Atualmente sua força de trabalho totaliza mais de 17.000 empregados, $85,9 \%$ baseados no Brasil e contribui para a geração de mais de 5.000 empregos indiretos (EMBRAER, 2006).

$\mathrm{O}$ desenvolvimento integrado do produto (DIP), conforme Sistema Normativo EMBRAER consiste em uma prática de execução de desenvolvimento de produtos que integra processos, planejamento integrado, organização e o uso de ferramentas adequadas.

Todas as decisões tomadas pelos indivíduos engajados no DIP devem ser fundamentadas nas virtudes que se desejam alcançar para o produto que está sendo desenvolvido, tais como custo, qualidade, cumprimento de prazos, logística e meio ambiente.

Dentro dos programas atuais, tem sido de extrema importância a participação conjunta da EMBRAER e das companhias parceiras selecionadas para desenvolver os segmentos e sistemas da aeronave. Assim sendo, as companhias parceiras são responsáveis por pacotes de trabalho, enquanto que a EMBRAER é tecnicamente responsável pelo avião como um todo, bem como pela integração de todos os sistemas e segmentos da aeronave.

Um Programa é a visão EMBRAER do conjunto de fases do ciclo-de-vida do produto sobre domínio, responsabilidade e interesse da empresa. O programa é representado em 5 fases: estudos de concepção e anteprojeto, definição conjunta, detalhamento e certificação, seriação e desativação (phase-out), planejadas e conduzidas em estágios apropriados, com análises críticas formais e documentadas, resultando em produtos especificados em cada uma delas.

A EMBRAER divide seus programas em três áreas:

a) aviação comercial: A EMBRAER continua a liderar o setor com suas inovadoras linhas de jatos regionais comerciais. Mais de 900 ERJs foram produzidos desde 1996 e entregues a mais de 30 companhias aéreas em 20 países. Oferecendo um alto índice de comunalidade entre os membros da família, os ERJ 135, ERJ 140 e ERJ 145, com disponibilidade para 37,44 e 50 passageiros respectivamente, oferecem versatilidade única para as empresas moldarem a capacidade das aeronaves com o tamanho do mercado. O jato de 70 assentos EMBRAER 170 é o primeiro de uma família de quatro aeronaves de última geração feitas para transportar entre 70 e 110 passageiros. O EMBRAER 175, de 78 lugares, fez seu vôo inaugural em junho de 2003 e foi certificado no quarto trimestre de 2004, enquanto que a campanha de certificação do EMBRAER 190, de 100 assentos, foi concluída no terceiro trimestre de 2005. O EMBRAER 195, com 108 lugares, foi certi- 
ficado em julho de 2006. Com comunalidade de até 95\% dentro da família, essas aeronaves têm cabines espaçosas, amplos compartimentos de bagagem e carga dianteiro e traseiro, além de desempenho superior.

b) aviação executiva: baseada na já testada plataforma do ERJ 135, a EMBRAER entrou no mercado da aviação executiva em dezembro de 2001 com a introdução do Legacy 600. A aeronave está disponível nas versões executive e shuttle, que se adaptam a uma variedade de aplicações mercadológicas, inclusive ao segmento de transporte de autoridades. Em maio de 2005, a EMBRAER anunciou o lançamento de dois novos jatos, Phenom 100 e Phenom 300 , nos segmentos very light e light. Além disso, em maio de 2006 foi anunciado o lançamento do Lineage 1000, no segmento ultra-large.

c) aviação defesa: a EMBRAER desempenha um papel estratégico no sistema de defesa brasileiro, tendo fornecido mais de $50 \%$ da frota da força aérea brasileira. Cerca de 20 forças aéreas no exterior também operam os produtos EMBRAER. Uma linha de produtos de defesa baseados na plataforma do ERJ 145, tais como o EMB 145 AEW\&C (airborne early warning and control), para alerta aéreo antecipado, o EMB 145 RS (remote sensing)/AGS (airborne ground surveillance), para sensoriamento remoto e o P-99, para patrulhamento marítimo e guerra anti-submarino, apresentam excelente potencial de vendas no concorrido mercado de defesa internacional. A EMBRAER já entregou à Força Aérea Brasileira (FAB), as oito aeronaves contratadas para o sistema de vigilância da amazônia, SIVAM. Outros produtos de sucesso destinados ao mercado incluem o Super Tucano, que em sua versão para a FAB, faz parte do Programa Sivam, sendo vetorado pelos EMB 145 AEW\&C. A EMBRAER concluiu em janeiro de 2005 as atividades de modernização do primeiro caça F-5 BR para a Força Aérea Brasileira (FAB).

De acordo com Yin (1994), um estudo de caso investigativo tem as seguintes características: lida com uma situação técnica distinta, em que haverá muitas mais variáveis práticas do que base de dados e como resultado; confia em fontes múltiplas como evidência; os dados necessitam convergir em uma forma de triangulação; benefícios do desenvolvimento prévio da proposição teórica para guiar o levantamento de dados e a análise.

O presente trabalho se enquadra nestas características, pois a avaliação do earned value será realizada por meio de processo investigativo com a realização de entrevistas, coleta de informações a partir de documentos e observações. Fundamentado em uma proposição teórica, previamente estabelecida, o presente trabalho pretende coletar diversas informações por meio de entrevistas com os principais envolvidos no processo de gestão do earned value e também da controladoria, via documental por intermédio de normas vigentes e de observações. Tal procedimento visa assegurar a confiabilidade dos dados coletados na avaliação de convergência entre as fontes. Busca-se na abordagem de pesquisa do tipo Estudo de Caso, analisar profundamente um ou mais objetos (casos), fazendo uso de múltiplos instrumentos de coleta de dados e intensa interação do pesquisador com o objeto de pesquisa. Portanto, um estudo de caso contempla a utilização conjunta de observação direta da realidade e lógica indutiva (BERTO; NAKANO, 1998).

Foi elaborado um protocolo de pesquisa, sobre custos, prazo e earned value (Quadro 2).

Além disso, foi desenvolvido um roteiro de entrevista para direcionar a coleta de dados realizada com os responsáveis, em cada Programa da EMBRAER, pela gestão do earned value. Com este roteiro pretende-se saber qual o conhecimento de cada um dos entrevistados sobre earned value, como eles aplicam a ferramenta, quais as possibilidades de melhoria e quais outras ferramentas de controle aplicadas.

Pela análise documental, identificaram-se as fases do Programa de Desenvolvimento de Produtos, planejadas e conduzidas em estágios apropriados, com análises críticas formais e documentadas, resultando em produtos especificados em cada uma delas.

As entrevistas foram realizadas durante 40 dias sem muita dificuldade, pois o pesquisador trabalha na empresa pesquisada. $\mathrm{O}$ tempo poderia ter sido ainda menor caso não fosse necessária à revisão de algumas respostas junto aos entrevistados. Foi percebida muita atenção dos entrevistados, pois o tema era de muito interesse para eles e a vontade de utilizar a ferramenta de maneira mais aplicada é muito grande.

Os entrevistados foram selecionados pelos seguintes parâmetros: ser o responsável dentro do planejamento de cada programa pela organização e disposição das informações necessárias para o cálculo do earned value e responder diretamente para as gerências; ter no mínimo 4 anos de tempo de trabalho na empresa; ter no mínimo um ano de trabalho em planejamento; possuir formação acadêmica com nível superior ( $3^{\circ}$ grau completo); ter conhecimentos em Gestão de Projetos, por meio de fontes formais (faculdades, instituições) ou informais (aprendizado em trabalho-“on job”). O Quadro 3 descreve o perfil dos entrevistados.

Todos os entrevistados atendem às características prédefinidas. Optou-se em utilizar um número limitado de entrevistados devido à abordagem qualitativa da pesquisa, obtendo-se um elevado detalhamento das questões abordadas. 
Quadro 2. Protocolo de pesquisa.

\begin{tabular}{|c|c|c|c|c|}
\hline \multirow{2}{*}{$\begin{array}{l}\text { Dimensão } \\
\text { abordada }\end{array}$} & \multirow[t]{2}{*}{ Questão de pesquisa } & \multicolumn{3}{|c|}{ Fonte } \\
\hline & & $\begin{array}{c}\text { Análise } \\
\text { documental }\end{array}$ & $\begin{array}{c}\text { Entrevista } \\
\text { semi-estuturada }\end{array}$ & Observação \\
\hline \multirow[t]{8}{*}{ Custos } & Qual sistema de custos é utilizado pela empresa? & $\mathrm{X}$ & & \\
\hline & Qual sua freqüência de contabilização? & $\mathrm{X}$ & & \\
\hline & É possível identificar com precisão os pagamentos antecipados? & $\mathrm{X}$ & & $X$ \\
\hline & É possível identificar com precisão os pagamentos postergados? & $\mathrm{X}$ & & $\mathrm{X}$ \\
\hline & $\begin{array}{l}\text { Como são controlados os orçamentos planejados X realizados } \\
\text { (geram ações corretivas de custos)? }\end{array}$ & $\mathrm{X}$ & & $\mathrm{X}$ \\
\hline & Como são orçados? & $X$ & $X$ & $X$ \\
\hline & Quando os orçamentos são atualizados? & $\mathrm{X}$ & $\mathrm{X}$ & $\mathrm{X}$ \\
\hline & $\begin{array}{l}\text { Quais são as atividades (etapas do DIP) X consumo das classes } \\
\text { de custos? }\end{array}$ & & $\mathrm{X}$ & $\mathrm{X}$ \\
\hline \multirow[t]{6}{*}{ Prazos } & Como são estabelecidos os prazos dos projetos? & $\mathrm{X}$ & & \\
\hline & Como são controlados os prazos dos projetos? & & $X$ & $X$ \\
\hline & $\begin{array}{l}\text { Quais são as atividades (fases do DIP) X prazos de execução } \\
\text { (faixas)? }\end{array}$ & $X$ & & \\
\hline & $\begin{array}{l}\text { Com que freqüência são atualizados os controles de prazo X } \\
\text { etapas do DIP? }\end{array}$ & $\mathrm{X}$ & & $\mathrm{X}$ \\
\hline & É possível identificar com precisão as atividades adiantadas? & & $\mathrm{X}$ & $\mathrm{X}$ \\
\hline & É possível identificar com precisão as atividades atrasadas? & & $\mathrm{X}$ & $\mathrm{X}$ \\
\hline \multirow[t]{10}{*}{ Earned value } & $\begin{array}{l}\text { Nível de conhecimento do entrevistado em earned value. O nível de } \\
\text { conhecimento foi avaliado por meio do questionamento: Explique } \\
\text { o que você entende sobre earned value como meio de controle de } \\
\text { projetos. }\end{array}$ & $\mathrm{X}$ & $X$ & \\
\hline & $\begin{array}{l}\text { Fale como você utiliza o earned value. Quais são os mecanismos } \\
\text { de incentivo? }\end{array}$ & & $\mathrm{X}$ & $\mathrm{X}$ \\
\hline & Qual sua opinião sobre o earned value? & & $\mathrm{X}$ & $\mathrm{X}$ \\
\hline & $\begin{array}{l}\text { Como você visualiza a utilização do earned value em } \\
\text { seu programa? }\end{array}$ & & $\mathrm{X}$ & $\mathrm{X}$ \\
\hline & Como você utiliza o earned value? & & $\mathrm{X}$ & $\mathrm{X}$ \\
\hline & Como o uso do earned value pode melhorar? & & $\mathrm{X}$ & $\mathrm{X}$ \\
\hline & $\begin{array}{l}\text { Que outras ferramentas você utiliza para controle? Em quais fases } \\
\text { do DIP elas se aplicam? }\end{array}$ & & $X$ & $\mathrm{X}$ \\
\hline & As ferramentas de controle atendem às suas expectativas? & & $\mathrm{X}$ & $\mathrm{X}$ \\
\hline & $\begin{array}{l}\text { Como você avalia cada uma das questões (mostrados os principais } \\
\text { fatores para uma boa aceitação do earned value descritos por Kim } \\
\text { et al. , 2003)? }\end{array}$ & & $\mathrm{X}$ & $\mathrm{X}$ \\
\hline & $\begin{array}{l}\text { Comente as barreiras para aplicabilidade do earned value } \\
\text { (descritos por THAMHAIN, 1998)? }\end{array}$ & & $X$ & $\mathrm{X}$ \\
\hline
\end{tabular}

Quadro 3. Características dos entrevistados.

\begin{tabular}{|c|c|c|c|c|c|c|}
\hline & \multicolumn{6}{|c|}{ Entrevistado } \\
\hline & 1 & 2 & 3 & 4 & 5 & 6 \\
\hline Tempo de EMBRAER (anos) & 20 & 5 & 6 & 6 & 17 & 4,5 \\
\hline Tempo na função (anos) & 1 & 2,5 & 6 & 6 & 17 & 4,5 \\
\hline Formação acadêmica & Engenharia & Engenharia & Administração & Engenharia & Engenharia & Administração \\
\hline $\begin{array}{l}\text { Formação em gerenciamento } \\
\text { de projetos }\end{array}$ & Não & Pós-graduação & Não & Pós-graduação & Certificado PMP & Não \\
\hline Áreas & & Executiva & & & ercial & Militar \\
\hline
\end{tabular}


As fases do Programa da EMBRAER consomem recursos que são alocados pelo sistema de custos. Considerando as classes de custos e buscando subsídios para a análise de aplicação do earned value, verificou-se nas observações, entrevistas e análise documental, a distribuição das classes ao longo das fases do programa. A distribuição descrita no Quadro 4 foi validada pelo funcionário entrevistado pela controladoria.

Verificou-se que o earned value é utilizado apenas na área de aviação militar, por ser uma exigência contratual e ter seu escopo bem definido em contrato. Muitas práticas do DIP da EMBRAER são descritas em procedimentos específicos. Todas as informações de custos estão alocadas no sistema de informação SAP que foi implantado em julho de 2006. O sistema SAP vem agregando todas as informações da EMBRAER de maneira sistematizada, eliminando os sistemas periféricos e paralelos de controle. Pretende-se que todas as informações da EMBRAER possam ser acessadas pelo SAP em suas bases de dados.

Os resultados das entrevistas e suas respectivas análises são sumarizados nos Quadros 5, 6 e 7.

A análise dos resultados (Quadro 4) permite identificar que a utilização do earned value é limitada devido à grande variação do escopo e à grande quantidade se fornecedores, com fluxos de caixa defasados (pagamentos em tempo diferente da execução do trabalho).

Dos dados coletados observou-se que os entrevistados das áreas de trabalho (aviação de defesa, executiva ou comercial) possuem um bom nível de conhecimento sobre o earned value. $\mathrm{O}$ entrevistado da área de aviação de defesa fez alguns comentários que acrescentaram ao trabalho: "Se o WBS for bem feito e o cronograma estiver em um nível adequado com um custo planejado adequado, pode-se ter earned value" e "Se houver mudança de escopo, deve existir uma alteração da linha de base para não corromper as estimativas".

As barreiras para aplicação do earned value foram analisadas conforme mostra o Quadro 6.

Uma análise geral do Quadro 6 permite identificar a aceitação por parte dos usuários e a necessidades de capacitação e adequação dos sistemas administrativos (SAP, apuração dos custos e prazos) para utilização do earned value. Segundo os entrevistados: “...existe um interesse por parte da gerência em utilizar o earned value, mas não chega a ser uma determinação...”; “...não sou incentivado a utilizar, apesar de conhecer a técnica."; "Na aviação de defesa, incentivamos e cobramos o uso do earned value.

Quadro 4. Distribuição dos custos nas fases do DIP na EMBRAER.

\begin{tabular}{llll}
\hline Fases do DIP na EMBRAER & & Classes de custo \\
\cline { 2 - 4 } & Mão de obra & Material & Muito baixo \\
Definições iniciais & Moderado & Muito baixo & Muito baixo \\
Definição conjunta & Moderado & Baixo & Muito baixo \\
Projeto detalhado e certificação & Alto & Muito alto & Moderado \\
Seriação & Alto & Baixo & Baixo \\
Phase out & Moderado & \\
\hline
\end{tabular}

Quadro 5. Análise do uso do earned value nas fases do programa de desenvolvimento de produtos da EMBRAER.

\begin{tabular}{lcl}
\hline \multicolumn{1}{c}{ Fase do DIP } & Utilização do EV & \\
\hline Definições iniciais & Difícil & Não existe um escopo definido. Na fase de definições, é estabelecido o escopo do \\
& & $\begin{array}{l}\text { projeto para as próximas fases e fica difícil estabelecer custos e prazos adequados. Os } \\
\text { custos e prazos dependem muito do andamento desta fase e podem sofrer variações } \\
\text { significativas. } \\
\text { Definição conjunta }\end{array}$ \\
Projeto detalhado & Elevada & $\begin{array}{l}\text { Escopo bem definido, prazos e necessidades de recursos (custos) estabelecidos e alo- } \\
\text { cados de maneira detalhada. }\end{array}$
\end{tabular}

Certificação

Seriação Difícil

Phase-out Difícil
Existe uma variação grande de escopo nas aeronaves que começam seu processo produtivo sem um cliente definido e também existem variações significativas de prazo devido ao atraso de matéria-prima e à falta de mão-de-obra em alguns casos. $\mathrm{O}$ envolvimento de vários fornecedores implicando em fluxos de caixa defasados. O único momento na fase de seriação que permite o uso do earned value é o controle das modificações de produto.

Existe uma variação grande de escopo. Todas as atividades são realizadas de acordo com o comportamento da frota, influenciando nos custos e prazos. Os valores podem ter variações expressivas tornando difícil um planejamento detalhado, pois todos os cálculos de garantias e retrofit são feitos com base em aeronaves já existentes. 
Ela é utilizada como uma ferramenta efetiva de gestão de projetos."; "É uma ferramenta que deveria ser aplicada, mas exige ser incorporada no dia-a-dia e termos dados confiáveis."; "É uma ferramenta muito útil, mas com pouca aplicabilidade na EMBRAER, pois nosso escopo tem muitas mudanças."

O Quadro 7 analisa os fatores principais para a boa aceitação do earned value.
O Quadro 7 identifica que existe aceitação para a utilização do earned value, mas fazem-se necessários mecanismos formais de disseminação e uso da técnica.

\section{Conclusões e recomendações}

A utilização do earned value como ferramenta de controle é incipiente na EMBRAER, pois o software que

Quadro 6. Barreiras para aplicabilidade do earned value no DIP da EMBRAER.

\begin{tabular}{|c|c|}
\hline $\begin{array}{c}\text { Barreiras para aplicabilidade do earned value } \\
\text { (THAMHAIN, 1998) }\end{array}$ & Análises sobre o uso do earned value na EMBRAER \\
\hline Falta de compreensão do funcionamento da técnica. & $\begin{array}{l}\text { Presente na EMBRAER. A compreensão da técnica é parcial, sendo significativa } \\
\text { apenas nos programas de defesa. }\end{array}$ \\
\hline Ansiedade quanto ao uso adequado da ferramenta. & Não foi identificada em nenhuma das entrevistas. \\
\hline $\begin{array}{l}\text { Utilização da ferramenta requerendo muito trabalho } \\
\text { e consumindo tempo. }\end{array}$ & $\begin{array}{l}\text { Identificou-se no segmento de defesa a existência de várias planilhas de coleta de } \\
\text { dados paralelas ao sistema de informação vigente (SAP). }\end{array}$ \\
\hline $\begin{array}{l}\text { Ferramentas limitando a criatividade no uso das es- } \\
\text { tratégias. }\end{array}$ & $\begin{array}{l}\text { Atualmente existe a possibilidade da incorporação de ferramentas de controle em } \\
\text { todos os programas, desde que aprovadas pela gerência e diretoria. }\end{array}$ \\
\hline $\begin{array}{l}\text { Inconsistência da ferramenta com os procedimentos } \\
\text { gerenciais/processos de negócios. }\end{array}$ & $\begin{array}{l}\text { O atual sistema de custos (custeio por absorção) é conciliado no último dia útil do } \\
\text { mês (só se obtém informações precisas dos custos no último dia útil do mês). A } \\
\text { existência de investimentos em TI que propiciarão acesso aos dados necessários } \\
\text { para o cálculo do EV. }\end{array}$ \\
\hline $\begin{array}{l}\text { Métodos de controle atuando como ameaçadores, no } \\
\text { que diz respeito à liberdade da equipe. }\end{array}$ & $\begin{array}{l}\text { Existem vários métodos de controle de custo e prazos, o EV foi identificado pelos } \\
\text { entrevistados como mais uma técnica de controle. }\end{array}$ \\
\hline $\begin{array}{l}\text { O propósito e seu benefício muitas vezes são vagos } \\
\text { e imprecisos. }\end{array}$ & $\begin{array}{l}\text { Os entrevistados reconhecem os benefícios auferidos com a aplicação do EV, po- } \\
\text { rém relataram que muitos colaboradores desconhecem a técnica. }\end{array}$ \\
\hline Custo de sua implementação considerado elevado. & $\begin{array}{l}\text { Não foram estimados os investimentos necessários para sua implementação, mas } \\
\text { os entrevistados relataram unanimemente que os investimentos não são restri- } \\
\text { ções. }\end{array}$ \\
\hline O trabalho não é conduzido em equipe; & Todos os programas da EMBRAER são conduzidos por equipe. \\
\hline $\begin{array}{l}\text { Equipe sempre ocupada em aprender novas ferra- } \\
\text { mentas. }\end{array}$ & $\begin{array}{l}\text { A limitação de tempo foi relatada como uma dificuldade devido ao momento em } \\
\text { que a EMBRAER se encontra atualmente (novos desenvolvimentos e carteira de } \\
\text { pedidos elevada). }\end{array}$ \\
\hline $\begin{array}{l}\text { Não atuando como ferramentas-controle e sim como } \\
\text { técnica justificadora de eventuais atrasos e desvios. }\end{array}$ & $\begin{array}{l}\text { Existe consenso entre os entrevistados de que o EV pode ser uma técnica com- } \\
\text { plementar. }\end{array}$ \\
\hline $\begin{array}{l}\text { Experiência anterior fracassada na utilização de ou- } \\
\text { tras técnicas. }\end{array}$ & $\begin{array}{l}\text { Não existem experiências de implantação nos segmentos de aviação executiva e } \\
\text { civil. }\end{array}$ \\
\hline Desconforto com a pouca familiaridade da técnica. & $\begin{array}{l}\text { É evidente a aceitação da técnica em algumas fases do DIP da EMBRAER (defi- } \\
\text { nição conjunta; projeto detalhado; certificação). }\end{array}$ \\
\hline
\end{tabular}

Quadro 7. Análise dos fatores para aceitação do EV no DIP da EMBRAER.

\section{Principais fatores para uma boa aceitação} do earned value (KIM et al., 2003)

A aceitação de earned value (EV) depende da aproximação Existe um apoio da alta gerência, porém não se identificaram mecanismos da alta gerência com a ferramenta. formais de incentivo ao uso do EV. A exceção é o segmento de aviação militar, em que o EV é exigido pelo cliente (força aérea dos EUA).

Estabelecimento de uma cultura voltada para projeto por $\mathrm{O}$ uso de gerenciamento de projetos encontra-se presente em todos os proparte dos gerentes funcionais e suas lideranças subsequientes gramas de maneira consistente e sistematizada.

para persuadir membros da equipe de projeto a usar o EV.

Flexibilidade aos gerentes funcionais e de projeto e aos tra- Os segmentos de aviação comercial e executiva não utilizam o EV. Porém balhadores ou grupos para selecionar seu próprio formulário existe a aceitação dos entrevistados em sua utilização, que identificam condo uso do earned value, dentro uma estrutura de diretrizes tribuições significativas do EV para o acompanhamento dos programas. gerais.

Fornecer o treinamento para os usuários do earned value. As capacitações acerca do EV são predominantemente individuais. Não foram identificadas evidências no plano de treinamento de sistematização para capacitações sobre o EV. 
permite a utilização de maneira integrada da ferramenta ainda não foi totalmente implementado e devido também à complexidade dos produtos desenvolvidos e à duração dos programas que ultrapassam 20 anos.

Pelo fato de o earned value não estar totalmente implantado, são baixas as exigências para a utilização da ferramenta e, como consequiência, existem variações no nível de conhecimento dos entrevistados e pouco treinamento institucionalizado.

Ficou evidente a grande utilização da ferramenta nos programas de defesa, por ser exigido em contrato. Devido a este fato, é necessário um grande esforço na atualização dos dados por meio de planilhas eletrônicas não integradas de maneira sistêmica. Mas as lições aprendidas nos programas de defesa podem ser utilizadas para ampliar o uso do earned value nas outras áreas.

Devido às características dos projetos, principalmente executivos e comerciais, fica difícil empregar o earned value em todas as fases do projeto.

A partir das evidências obtidas nos dados coletados, identifica-se que o earned value é uma ferramenta de controle que vem ganhando aceitação entre os entrevistados.

A implementação do earned value não é fácil e necessita do empenho da alta gerência, investimentos em ferramentas de TI e treinamento.

Para uma boa utilização do earned value, é fundamental se ter o escopo do projeto muito bem definido, um cronograma detalhado e custos previstos das atividades em um nível adequado, para se medir o avanço físico e obter valores confiáveis do earned value.

Vale lembrar que as fases, nas quais a utilização da ferramenta é difícil, estão relacionadas aos processos da empresa e não da técnica. Outro problema verificado foi que a atualização dos dados no earned value só pode ser realizada uma vez por mês, por causa do ciclo contábil da empresa (fechamento mensal), pois as apurações dos dados se tornam imprecisas quanto mais longe do fechamento contábil estiverem. Com relação a prazo, não existem muitos problemas, depende apenas da disposição das pessoas em atualizar os cronogramas.

Ficou evidente que o earned value pode ser parcialmente aplicada e não necessariamente uma técnica a ser utilizada ou não (questão binária), como é abordado na literatura.

A implantação do earned value seria de grande valia para a EMBRAER, pois contribui no controle do processo de desenvolvimento de produtos (definição conjunta, projeto detalhado, certificação e também nas modificações) integrando as áreas (engenharia, programa, contratos, suprimentos e produção) e auxiliando no cumprimento de prazos e custos, o que assegura o sucesso do projeto.

É possível o uso do earned value na EMBRAER, porém é necessário: realizar treinamento maciço para os usuários do earned value; obter envolvimento e suporte da alta gerência, especialmente o fornecimento de recursos suficientes e a promoção de seu uso; fornecer capacitação técnica e administrativa aos gerentes de projeto; utilizar a base de dados eletrônica, preferencialmente a já existente; propiciar e garantir o uso do earned value como parte de um sistema de gerência integrado de projeto; manter as ações de aperfeiçoamento da base de dados eletrônica; e criar uma equipe composta por membros de todos os programas com o intuito de disseminar as boas práticas e as troca de experiências.

Como sugestão para a continuidade desta pesquisa, propõe-se: desenvolver uma sistemática para a consolidação do uso do earned value na EMBRAER; analisar a adequação dos sistemas de custeio em relação às necessidades de controle do gerenciamento de projetos complexos; analisar os sistemas de controle e gestão aplicados nos Programas de desenvolvimento de produtos na EMBRAER. 


\title{
On the use of the earned value management system approach for complex projects in the brazilian aircraft industry
}

\begin{abstract}
Earned value management has been increasingly used by companies as well as by functional and project managers due to its effectiveness in controlling project results. It is defined as a control tool that enables a quantitative and simultaneous evaluation of the costs and process time within a pre-scheduled date as well as predicting the deadline and the total value spent (VARGAS, 2005).This study evaluates the earned value applicability as a tool to control aeronautical projects at EMBRAER, A Brazilian Aeronautical Company. It is also concerned with the application of earned value in the control of complex projects. The data were gathered through interviews, observations and document analysis. The results show that The Earned Value is used only in the defense aviation projects since it is a contractual obligation. Its use in executive and commercial areas is restricted to the combined definition stages, detailed project, and certification because the scope of the project is well defined, the schedule is detailed, and the costs are foreseen and fairly accurate. In the other stages of the product development, the use of earned value is restricted mainly because of the frequent changes of the scope of the product and project.
\end{abstract}

Keywords: Project. Control. Earned value.

\section{Referências bibliográficas}

BERTO, R. M. V. S.; NAKANO, D. N. Métodos de Pesquisa na Engenharia de Produção. In: ENCONTRO NACIONAL DE ENGENHARIA DE PRODUÇÃO, XVIII, 1998, Niterói. Anais... Niterói: UFF/ABEPRO, 1998. CD-ROM.

BOWMAN, R. A. Developing activity duration specification limits for effective project control. European Journal of Operational Research, v. 174, n. 2, p. 1191-1204, 16 oct. 2006.

CIOFFI, D. F. Designing project management: A scientific notation and an improvement formalism for Earned Value calculations. International Journal of Project Management, v. 24, n. 2, p. 136-144, 2006.

FLEMING, Q. W.; KOPPELMAN, J. M. Earned Value Project Management. 2. ed. Newton Square, Philadelphia: Project Management Institute, 2000.

GOMES, W.O. Gestão de Projetos: Proposta de Modelo para Implantação em Organização Híbrida com Estrutura Matricial Leve. Dissertação (Mestrado Profissional) - Universidade de Campinas. Engenharia Mecânica, 2004.

KWAK, Y. H.; WATSON, R. J. Conceptual estimating tool for technology-driven projects: exploring parametric estimating technique. Technovation, v. 25, n. 12, p. 1430-1436, 2004.

KERZNER, H. Project Management - A systems approach to palnning, scheduling and controlling. 8. ed. New Jersey: John Wiley \& Sons, Inc., Hoboken, 2005.

Gestão de Projetos: As Melhores Práticas. 2. ed. São Paulo: Bookman, 2006.

KIM, E. H.; WILLIAM, G. W. Jr; MICHAEL, R. D. A model for effective implementation of Earned Value Management methodology. International Journal of Project Management, v. 21, n. 5, p.375-382, 2003.

KIM, E. H. A Study on the $\mathbf{E}$ ffectiv e Imp lementation of Earned value Management Methodology. Unpublished doctoral dissertation. The George Washington University, Washington, DC, 2000 .

LEE, S. H.; PEÑA-MORA, F.; PARK, M. Dynamic planning and control methodology for strategic and operational construction project management. Automation in Construction, v. 15, n. 1, p. 84-97, 2005.

MARTINS, E. Contabilidade de Custos. 8. ed. São Paulo: Ed. Atlas, 2001.

NAVON, R. Automated project performance control of construction projects. Automation in Construction, v. 14, n. 4, p. 467-476, 2005.

OLIVEIRA, R. C. F. Gerenciamento de Projetos e a Aplicação da Análise de Earned Value em Grandes projetos. Dissertação (Mestrado) - Escola Politécnica da Universidade de São Paulo. Departamento de Engenharia Naval e Oceânica, 2003.

PMBoK. Conjunto de Conhecimentos em Gerenciamento de Projetos. 3. ed. Four Campus Boulevard, Newtown Square PA: Project Management Institute, 2004.

ROVAI, R. L.; TOLEDO, N. N. Avaliação de Performance de Projetos Através do Earned Value Management System. In: ENCONTRO NACIONAL DE ENGENHARIA DE PRODUÇÃO, XXII., 2002, Curitiba. Anais... Curitiba: ABEPRO, 2002. CD-ROM.

ROZENES, S.; VITNER, G.; SPRAGGETT, S. MPCS: Multidimensional Project Control System. International Journal of Project Management, v. 22, n. 2, p.109-118, 2004.

SHENHAR, A. J.; WIDEMAN R. M. Optimizing Project Success by Matching PM Style with Project Type. Project Management Forum, 2000. Disponível em: <http://scholar. google.com/scholar?q=Optimizing+Project + Success + by $+M$ atching + PM\&hl=pt-BR\&um $=1 \& \mathrm{ie}=\mathrm{UTF}-8 \& \mathrm{oi}=$ scholart $>$. Acesso em: 18 jan. de 2006.

THAMHAIN, H. J. Integrating Project Management Tools with the Project team. In: ANNUAL PROJECT MANAGEMENT INSTITUTE SEMINARS \& SYMPOSIUM, 29., 1998., Long Beach

VARGAS, R. Análise de Earned Value. 3. ed. São Paulo: Brasport, 2005. 
VÄLIMAÄKI, J. True Project management is Earned Value based cost management. Project Management, v. 3, n. 1, p. 8-9, 1995

WILliAMS, T. M. Modelling Complex Projects. New York: John Wiley \& Sons, Ltd, 2002.
YIN, R. K. Case Study Research Desing and Methods. Thousand Oaks: SAGE Publications, Inc, 1994.

$<$ http://www.EMBRAER.com.br/portugues/content/empresa/ profile.asp> sítio da EMBRAER. Acesso em: 22 fev. 2006.

\section{Sobre os autores}

\section{Rogério Abdala Giacometti Carlos Eduardo Sanches da Silva}

Grupo de Pesquisa em Qualidade e Produto, Instituto de Engenharia de Produção e Gestão, Universidade Federal de Itajubá - UNIFEI CEP 37500-903, Caixa Postal 150, Itajubá, MG, Brasil,

e-mails: giacometti@embraer.com.br; sanches@unifei.edu.br

\section{Helder José Celani de Souza}

\section{Fernando Augusto Silva Marins}

Grupo de Otimização e Logística - GOL, Departamento de Engenharia de Produção, Campus de Guaratinguetá, Universidade Estadual Paulista - UNESP, SP, Brasil, e-mails: hcelani@uol.com.br; fmarins@feg.unesp.br

\section{Elizabete Ribeiro Sanches da Silva}

Faculdade de Ciências Sociais Aplicadas do Sul de Minas, Caixa Postal 499, CEP 37504-066, Itajubá, MG, Brasil, e-mail: beribeiro@uol.com.br

Agradecimentos: À EMBRAER pela oportunidade do desenvolvimento desta pesquisa e, em especial, aos entrevistados. À FAPEMIG e CAPES pela disponibilização de recursos financeiros obtidos através dos projetos de fomento em desenvolvimento.

Recebido em 05/6/07 Aceito em 28/11/07 
